\title{
XVI. Ueber die Krystallformen des tetra- und des pentathionsauren Kaliums.
}

\author{
Von
}

\author{
A. Fock in Berlin.
}

(Mit 3 Holzschnitten.)

Bekanntlich giebt es im monosymmetrischen Systeme zwei mögliche Unterabtheilungen, die hemimorphe Gruppe und die hemiëdrische Gruppe*). Bei der ersteren besteht keine Symmetrieebene mehr, wohl aber noch eine Symmetrieaxe; bei der zweiten ist dagegen die Symmetrieaxe verschwunden, während die Symmetrieebene fortexistirt.

Bisber kannte man nur Substanzen, welche der he-

Fig. 1.

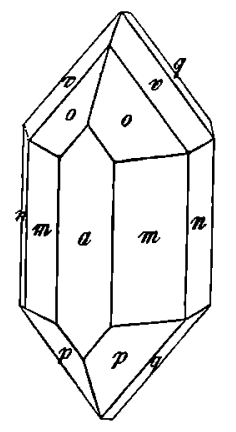
mimorphen Abtheilung angehören; ich glaube indessen in dem tetrathionsauren Kalium eine Substanz aufgefunden zu baben, welche der zweiten Abtheilung, der hemiëdrischen Gruppe, zuzuweisen ist.

Das tetrathionsaure Kalium ist bereits vor einer Reihe von Jahren von Rammelsberg, aber unter falschem Namen und falscher Formel, nämlich als pentathionsaures Kalium**) beschrieben worden. In den Angaben des genannten Forschers findet sich indessen keinerlei Bemerkung, welche auf eine Hemiëdrie hinweist und auch die beigegebene Figur (s. Fig. 1) zeigt einen vollständig holoëdrischen Charakter.

Nachstehend finden sich zunächst die Angaben R a mmel sb er g's vollständig wiedergegeben, da in denselben mehrere kleine Rechenfehler aufgefunden wurden. Die letzteren sind hier berichtigt worden.

*) Li ebi sch, Physik. Krystallographie. Leipzig 1894, 49.

**) R a m mels berg, Handbuch der kryst. Chemie 1, 495 ; vergl. hierüber Ber. d. d. chem. Ges. $23,9428$. 


$$
\begin{gathered}
a: b: c=0,9285: 1: 1,2642 \\
\beta=78^{\circ} 28^{\prime} .
\end{gathered}
$$

Beobachtete Formen : $a=\{100\} \infty P \infty, o=\{111\}-P, p=\left\{T_{11}\right\}+P$, $m=\{110\} \infty P, \quad n=\{130\} \infty R 3, \quad c=\{\bar{T} 03\}+\frac{1}{3} p_{\infty}, \quad v=\{133\}-R 3$, $q=\{\bar{T} 33\}+R 3$.

Beobachtet: Berechnet:

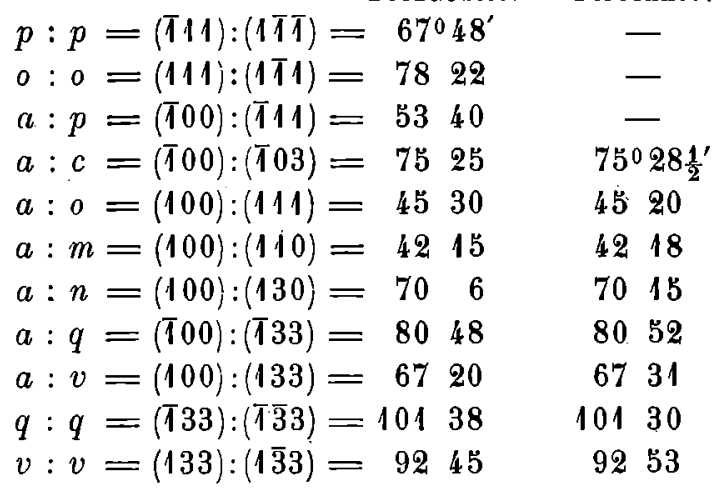

R ammelsberg giebt ferner an, dass die Krystalle "stets mit einem $a$ aufgewachsen, welches sehr vorherrschl «.

Die von mir untersuchten Krystalle waren wasserfrei und zeigten die Zusammensetzung $K_{2} S_{4} O_{6}$. Nachstehend finden sich die erhaltenen Messungsresultate wiedergegeben. Die Aufstellung der Krystalle wurde aus leicht ersichtlichen Gründen etwas anders gewählt. Das Hemidoma $c\{\bar{T} 03\}$ wurde zur Basis $\{001\}$, die prismatischen Formen dagegen unverändert beibehalten.

$$
\begin{gathered}
a: b: c=0,93017: 1: 1,26664 \\
\beta=75^{044^{\prime}} .
\end{gathered}
$$

Beobachtete Formen: $a=\{100\} \infty P \infty, c=\{001\} 0 P$, $q=\{011\} R \infty, m=\{110\} \infty P, \quad p=\{111\}-P, \quad o=$ $\{111\}+P, v=\{\overline{133}\}+R 3$.

Die Krystalle sind tafelförmig nach dem Orthopinakoid $a$ und an dem einen Ende der Verticalaxe gänzlich anders ausgebildet als an dem anderen. An dem einen Ende erscheint regelmässig die Basis $c$ mit dem Klinodoma $q$, an dem anderen Ende beobachtet man dagegen bisweilen die Pyramide $v\{133\}$ allein, meistens daneben auch $o\{T 11\}$ und vielfach auch $p\{111\}$ (s. Fig. 2). In seltenen Fällen findet sich an dem letzteren Ende noch das Fig. 9.

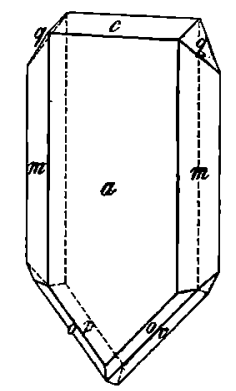
Klinodoma $q$ ausgebildet, indessen in ganz untergeordneter Ausdehnung, und ebenso trifft man ausnahmsweise an dem ersteren Ende auch wobl die 
Pyramide 0 . Das Prisma $m\{110\}$ tritt regelmässig mit allen vier Flächen auf, das Klinoprisma $n\{130\}$ wurde nicht beobachtet.

Einzelne wenige Individuen sind scheinbar holoëdrisch ausgebildet, indem die Basis gegenüber dem Klinodoma zurücktritt oder gänzlich fehlt.

\begin{tabular}{|c|c|c|}
\hline \multicolumn{2}{|c|}{ Beobachtet: } & Berechnet: \\
\hline$a: n$ & $i=(100):(110)=4202$ & - \\
\hline$a: c$ & $=(100):(001)=7544$ & - \\
\hline$q: q$ & $=(011):(0 \bar{T} 1)=101 \quad 40$ & - \\
\hline$a: q$ & $=(100):(011)=81 \quad 7$ & $84^{0} 3^{\prime}$ \\
\hline$m: q$ & $=(110):(011)=5041$ & 5036 \\
\hline$m: q$ & $=(T 10):(011)=6613$ & $66 \quad 12$ \\
\hline$a: p$ & $=(100):(111)=453$ & $44 \quad 12$ \\
\hline$a: o$ & $=(\bar{T} 00):(\bar{T} 11)=5336$ & $54 \quad 30 \frac{1}{2}$ \\
\hline$a: v$ & $=(\bar{T} 00):(\bar{T} 33)=678$ & 6553 \\
\hline$v: v$ & $=(\overline{1} 33):(\overline{1} \overline{3} 3)=9219$ & 9130 \\
\hline$p: p$ & $=(111):(1 \pi 1)=6750$ & 6621 \\
\hline $0: 0$ & $=(\bar{T} 11):(\bar{T} T 1)=7831$ & 7926 \\
\hline
\end{tabular}

Spaltbarkeit ziemlich vollkommen nach dem Orthopinakoid $a$.

Ebene der optischen Axen = Symmetrieebene.

Durch das Orthopinakoid tritt eine Axe aus und zwar im spitzen Winkel $\beta$, scheinbar circa $48^{\circ}$ gegen die zugehörige Normale geneigt.

Ein Vergleich der beiden Messungsresultate zeigt, dass an der völligen Identität der betreffenden Krystalle bezw. Substanzen nicht im Geringsten zu zweifeln ist. Die von Rammelsberg gegebene Zeichnung (Fig. 1) giebt zwar nur holoëdrische Formen wieder, die Krystalle eines von demselben herrübrenden Originalpräparates zeigten indessen genau die hemiëdrische Ausbildung der Fig. 2.

Auch Baker, der das telrathionsaure Kalium auf Grund einer mikroskopischen Untersuchung beschreibt und gleichfalls den Austritt einer Axe durch das Orthopinakoid beobachtete, kennzeichnet die Krystalle als hemimorph. Wenn er die Substanz für rhombisch hält, so ist das wohl nur dem Unstande zuzuschreiben, dass er keine Messungen anstellte und eine derartige Hemiëdrie, wie sie hier vorliegt, im monosymmetrischen Systeme bisher nicht bekannt war und zum Theil auch wohl nicht für möglich gehalten wurde.

Bemerkenswerth bleibt aber die grosse Differenz, welche sich hinsichtlich gewisser Winkel kund giebt. Die von Bammelsberg angefübrten beobachteten und berechneten Winkel zeigen zwar eine recht befriedigende

*) Journ. of the Chem. Soc. 43, 354 . 
Uebereinstimmung; leider war es mir nicht möglich, trotzdem gut ausgebildete Krystalle vorlagen, das gleiche Resultat zu erzielen.

Bei den von mir untersuchten verschiedenen Präparaten (ebenso bei dem untersuchten Rammelsberg'schen Originalpräparate) waren regelmässig die Flächen der Krystalle an dem Ende der Verticalaxe am vollkommensten ausgebildet, an welchem die Basis erscheint, und soweit diese Flächen in Betracht kommen, lässt die Uebereinstimmung zwischen den berechneten und beobachteten Winkeln nichts zu wunschen übrig; sie stellt sich erheblich grösser als bei Rammelsberg. Geht man aber zu den Flächen des anderen Poles uber, so macht sich eine auffällige Differenz bemerkbar. Wie weit dieselbe eine zufällige ist, lässt sich einstweilen nicht entscheiden. Obwohl eine grosse Anzahl von Krystallen gemessen wurde, konnte doch eine nähere Gesetzmässigkeit in dieser Beziehung nicht aufgefunden werden. Vielleicht ist jene Diflerenz bezw. das Schwanken des anderen Poles nicht ohne einen gewissen Zusammenhang mit der eigenthümlichen Hemiëdrie des Systemes.

Das pentathionsaure Kalium ist bisher krystallographisch noch nicht beschrieben worden. Dasselbe wird neben dem tetrathionsauren Kalium erhalten, wenn man die Wackenroder'sche Flussigkeit mit essigsaurem Kalium behandelt, es krystallisirt dann zuletzt aus und zwar mit $1 \frac{1}{2}$ Molekülen Krystallwasser ${ }^{*}$ ). Die Formel lautet $2 \mathrm{~K}_{2} \mathrm{~S}_{5} \mathrm{O}_{6}+3 \mathrm{H}_{2} \mathrm{O}$. Die krystallographische Untersuchung ergab Folgendes.

Krystallsystem : Rhombis ch.

$$
a: b: c=0,4564: 1: 0,3051 \text {. }
$$

Beobachtete Formen : $b=\{010\} \infty \breve{P} \infty, c=\{001\} 0 P, m=\{110\} \infty P$, $n=\{130\} \infty \breve{P} 3, q=\{011\} \breve{P} \infty, p=\{221\} 2 P, o=\{241\} 4 \breve{P} 2$.

Die farblosen Krystalle sind meist prismatisch nach der Verticalaxe und bis $2 \frac{1}{2} \mathrm{~mm}$ lang und $1 \mathrm{~mm}$ dick. Von den Flächen der Prismenzone herrschen diejenigen des primären Prismas stets vor. Das Brachypinakoid erscheint regelmässig untergeordnet, während das Prisma mit dreifacher Brachyaxe nur ganz vereinzelt in minimaler Ausdehnung auftritt. Von den Endflächen sind meist diejenigen des Domas am grössten ausgebîdet, nicht selten zeigen aber auch die Flächen der Pyramiden die gleiche Ausdehnung. Die Basis $c$ wurde nur an zwei lndividuen beobachtet (s. Fig. 3).

Fig. 3.

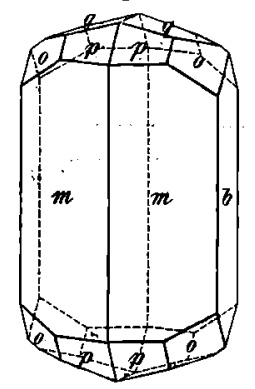

*) Ber. d. d. chem. Ges. 23, 2431. 
240 A. Fock. Ueber die Krystallformen des tetra- u. des pentathionsauren Kaliums.

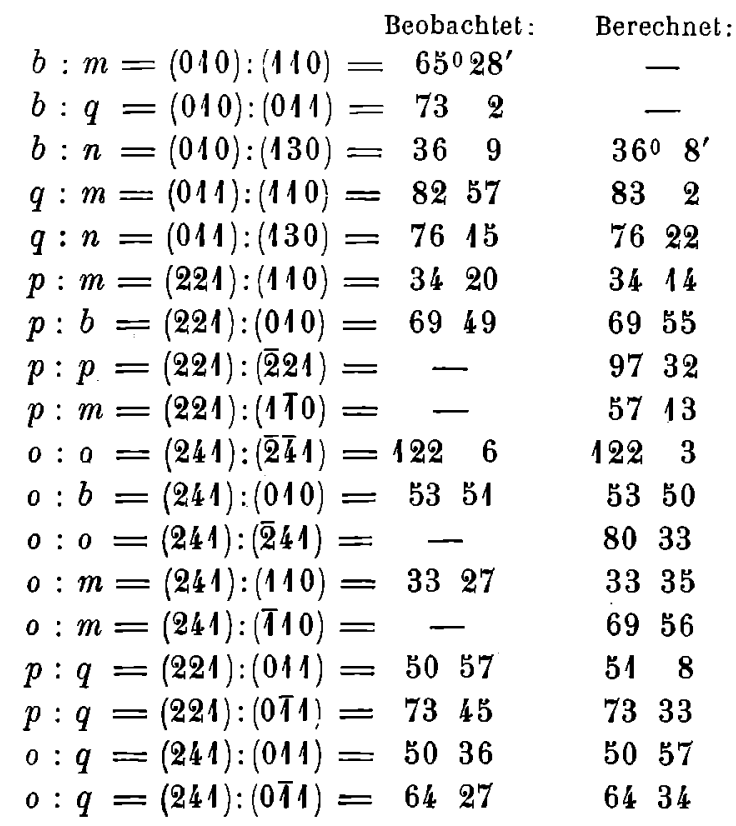

Spaltbarkeit nicht beobachtet.

Für eine nähere optische Untersuchung waren die Krystalle zu trube. Später erhaltene Präparate bestanden zum Theil aus Krystallen, welche dicktafelförmig nach dem Brachypinakoid waren. Als Randflächen wurden an denselben vielfach einzig die Flächen der Pyramide $p\{221\}$ beobachtet, nicht selten zeigten sich auch noch weitere Formen, doch traten dieselben der Ausdehnung nach mehr zurúck. Neue Formen konnten an diesen Präparaten nicht aufgefunden werden.

Baker hat schon fruher das pentathionsaure Kalium in Händen gebabt, indessen nur auf Grund einer mikroskopischen Untersuchung beschrieben*). Er charakterisirt die Krystalle als dicke Prismen. Die Individuen des betreffenden Präparates durften demnach die zuerst beschriebene Ausbildung besessen haben.

*) Journ. of the Chem. Soc. 43,354 . 